\title{
Madame Dunoyer, Lettres historiques et galantes de deux dames de condition dont l'une était à Paris et l'autre en Province
}

\section{Antonella Amatuzzi}

\section{(2) OpenEdition \\ 1 Journals}

\section{Edizione digitale}

URL: https://journals.openedition.org/studifrancesi/3070

DOI: $10.4000 /$ studifrancesi.3070

ISSN: 2421-5856

\section{Editore}

Rosenberg \& Sellier

\section{Edizione cartacea}

Data di pubblicazione: 1 juillet 2013

Paginazione: 450-451

ISSN: 0039-2944

\section{Notizia bibliografica digitale}

Antonella Amatuzzi, «Madame Dunoyer, Lettres historiques et galantes de deux dames de condition dont l'une était à Paris et l'autre en Province», Studi Francesi [Online], 170 (LVII | II) | 2013, online dal 30 novembre 2015, consultato il 02 février 2023. URL: http://journals.openedition.org/studifrancesi/3070 ; DOl: https://doi.org/10.4000/studifrancesi.3070

Questo documento è stato generato automaticamente il 2 février 2023.

\section{c) (†) $\odot$}

Creative Commons - Attribuzione - Non commerciale - Non opere derivate 4.0 Internazionale - CC BYNC-ND 4.0

https://creativecommons.org/licenses/by-nc-nd/4.0/ 


\title{
Madame Dunoyer, Lettres historiques et galantes de deux dames de condition dont l'une était à Paris et l'autre en Province
}

\author{
Antonella Amatuzzi
}

\section{NOTIZIA}

MADAME DUNOYER, Lettres historiques et galantes de deux dames de condition dont l'une était à Paris et l'autre en Province, Texte présenté et annoté par Nancy o'conNoR, Nouvelle édition basée sur celle de Pierre Marteau [Pierre Husson] Cologne [La Haye] 1733 (5 volumes), Rennes, Presses Universitaires de Rennes, 2012, pp. 431.

$1 \quad$ Les Lettres historiques et galantes furono un grande successo editoriale del Settecento. Si tratta della corrispondenza fittizia tra due amiche, una residente a Parigi e l'altra che viaggia nel Midi e poi all'estero (Aquisgrana, Utrecht, L'Aia) il cui primo tomo è stato pubblicato nel 1707. L'autrice è Anne Marguerite Petit Dunoyer (1663-1719), originaria di una famiglia della borghesia protestante di Nîmes, orfana, cresciuta dalla zia materna Marguerite Cotton Saporta à Orange, trasferitasi dopo la revoca dell'Editto di Nantes a Ginevra e poi nelle Province Unite. Sposatasi nel 1688 col cattolico Guillaume Dunoyer, capitano del reggimento di Tolosa, rinuncia alla sua fede ma nel 1701 lo lascia e si stabilisce all'Aia, dove diventa una delle prime donne giornaliste.

2 I temi trattati, in parte autobiografici (nella dama di provincia è impossibile non scorgere alcune contiguità con la stessa Madame Dunoyer), sono vari e comprendono aneddoti divertenti, notizie scandalose della Corte e della provincia, resoconti di avvenimenti storici, critica sociale e politica, informazioni e dettagli sulla architettura e le abitudini delle città visitate, questioni religiose (in particolare riflessioni contro l'ipocrisia dei devoti e la superstizione e ferma denuncia dell'intolleranza). Secondo la 
moda del 'mélange des genres', caratteristica della pratica letteraria dell'epoca, vengono inserite anche novelle, canzoni, poesie.

3 Le lettere si caratterizzano per il tono disinvolto e libero, a volte audace, anche grazie al fatto di essere pubblicate all'Aia e quindi al riparo da censure e condanne. Lo stile è naturale e spigliato, elegante e senza 'affectation' (p. 18).

4 Come scrive la curatrice dell'edizione nell'introduzione (che occupa le pp. 9-29) «l'originalité de l'ouvrage pour la forme, le fond et le ton vient en définitive d'une stratégie d'écriture destinée à faire du lecteur à la fois le confident privilégié et le complice des correspondantes, du rapport complexe qu'on y trouve entre histoire et fiction et de ce que les Lettres historiques et galantes fondent un nouveau genre qu'on pourrait appeler journalisme épistolaire» (p. 10).

5 Il valore delle Lettres è rilevante soprattutto dal punto di vista storico in quanto rappresentano una testimonianza della vita in Francia alla fine del regno di Luigi XIV e all'inizio della reggenza e annunciano, con la loro libertà e irriverenza, il clima critico che pervaderà il secolo dei Lumi.

6 Il testo scelto per la presente edizione è quello del 1733, qui modernizzato nell'ortografia e nella punteggiatura e corredato da note esplicative che permettono di identificare i personaggi citati e di chiarire alcune allusioni.

7 Completano il volume una tavola cronologica, un indice dei nomi e una ricca bibliografia. 